\title{
Mott insulators in an optical lattice with high filling factors
}

\author{
D. van Oosten, ${ }^{1,2}$ P. van der Straten, ${ }^{2}$ and H. T. C. Stoof ${ }^{1}$ \\ ${ }^{1}$ Institute for Theoretical Physics, Utrecht University, Leuvenlaan 4, 3584 CE Utrecht, The Netherlands \\ ${ }^{2}$ Debye Institute, Utrecht University, Princetonplein 5, 3584 CC Utrecht, The Netherlands
}

(Received 3 May 2002; published 21 March 2003)

\begin{abstract}
We discuss the superfluid to Mott insulator transition of an atomic Bose gas in an optical lattice with high filling factors. We show that in this multiband situation, the long-wavelength physics is described by a singleband Bose-Hubbard model. We determine the many-body renormalization of the tunneling and interaction parameters in the effective Bose-Hubbard Hamiltonian, and consider the resulting model at nonzero temperatures. We show that, in particular, for a one- or two-dimensional optical lattice, the Mott-insulator phase is more difficult to realize than anticipated previously.
\end{abstract}

DOI: 10.1103/PhysRevA.67.033606

PACS number(s): 03.75.Hh, 67.40.-w, 32.80.Pj, 39.25.+k

\section{INTRODUCTION}

The behavior of trapped Bose-Einstein condensates offers a large amount of interesting features. Specifically, the phase coherence of a condensate creates the prospect of various interference experiments, as shown for the first time in an experiment performed by Andrews et al. [1]. In another experiment, a large number of condensates trapped in a periodic lattice potential have been made to interfere [2]. Having shown experimentally that, in general, condensates are phase coherent and thus show off-diagonal long-range order, the question arises whether this long-range order can also be destroyed in a controllable way. This was only very recently achieved in a beautiful experiment by Greiner et al. [3].

In this last experiment, a trapped Bose-Einstein condensate is put into a three-dimensional optical lattice. The number of atoms in the condensate is sufficiently large to obtain a filling factor of almost two atoms per site. By increasing the intensity of the lattice light, a quantum phase transition from a superfluid state to a Mott insulating state is achieved. In the insulating phase, all phase coherence is lost due to quantum fluctuations. The transition was predicted to occur in this system by Jaksch et al. [4], and the observed critical conditions for the transition are in good agreement with the results of an on-site mean-field theory [5]. This indicates that the single-band Bose-Hubbard model used in Refs. [4,5] can accurately describe a gas of ultracold bosonic atoms in an optical lattice when the filling factor of the lattice is of the order of 1 .

However, this model is no longer valid in the case of higher filling factors such as described in the experiments of Refs. [6,7]. The theories mentioned above use single-particle wave functions corresponding to the lowest band of the lattice to calculate the microscopic parameters of the singleband Bose-Hubbard model as a function of the lattice parameters. In the case of high filling factors more than one band is generally populated, leading to a multiband Bose-Hubbard model. The interaction effects that occur under these circumstances have not been considered previously. Furthermore, the effects of thermal fluctuations are also not understood, even in the single-band Bose-Hubbard model. There are studies that describe number squeezing in an optical lattice at nonzero temperature $[8,9]$, but they are not in the strongly interacting limit that is relevant for the Mott insulator. Our main objective here is to develop an effective theory that can deal with these issues.

\section{SOLVING THE MULTIBAND BOSE-HUBBARD MODEL}

To solve the problems associated with high filling factors, we have to deal with the many-body physics at every site. Since the high filling factors of interest are experimentally most relevant in low-dimensional lattices [6,7], we discuss the energy scales involved in those systems. In a lowdimensional lattice, we can approximate the on-site trapping potential by an anisotropic harmonic potential. The oscillator frequencies $\omega_{\|}$and $\omega_{\perp}$ correspond to the trapping frequencies in the directions parallel and perpendicular to the periodicity of the lattice, respectively. Because the typical size of a lattice well in the parallel direction(s) is much smaller than in the perpendicular direction(s), we immediately have that $\hbar \omega_{\perp} \ll \hbar \omega_{\|}$. Furthermore, for the experimental conditions of interest $[6,7]$, the temperature is in between the two trapping frequencies, i.e., $\hbar \omega_{\perp} \ll k_{B} T \ll \hbar \omega_{\|}$. This implies that in every site the gas is in the parallel direction(s) in the ground state of the potential, but that it occupies many states in the perpendicular direction(s). In particular, this holds for the thermal cloud of the gas. As a consequence, the effective dimensionality of the gas at every site is reduced and the thermal excitations are only present in the perpendicular direction(s). It is under these conditions that we are able to solve the relevant multiband Bose-Hubbard model by using the following two-step procedure.

First, we solve the many-body physics at every site. Due to the famous infrared problems of a one- or twodimensional Bose gas this is not an easy task, and an accurate equation of state for these gases was developed only very recently in the weakly interacting limit [11]. This equation of state is found by treating phase fluctuations in the (quasi)condensate exactly and we can, in particular, use it to determine at every temperature the number of atoms in the (quasi)condensate $N_{0}(T)$. Furthermore, it is shown in Ref. [11] that even in the presence of phase fluctuations, the Gross-Pitaevskii equation can still be used to calculate the density profile of the (quasi)condensate.

Second, we consider the coupling between the sites. Since 
we have a (quasi)condensate at every site, the coupling between sites will be dominated by tunneling from (quasi)condensate to (quasi)condensate as opposed to (quasi)condensate to thermal cloud. This means that we can describe the coupling between sites by a single-band Bose-Hubbard model. The important parameters in the Bose-Hubbard model are the on-site interaction energy $U$ and the energy $t$ associated with the tunneling of atoms between nearestneighbor sites. Both energies can be calculated from the knowledge of the (quasi)condensate wave function $\psi_{0}(\mathbf{x})$ $=\sqrt{n_{0}(\mathbf{x})} e^{i \vartheta}$, where $n_{0}(\mathbf{x})$ is the density profile and $\vartheta$ is the global phase of the (quasi)condensate. The interaction energy $U$ is proportional to $\int d \mathbf{x}\left|\psi_{0}(\mathbf{x})\right|^{4}$, whereas the tunneling energy $t$ requires the evaluation of an overlap integral between the (quasi)condensate wave functions of two neighboring sites in the parallel direction(s).

To describe the effect of the interatomic interaction, we thus need to determine how the (quasi)condensate wave function changes as a result of the on-site interactions. Since the mean-field interaction obeys $\hbar \omega_{\perp} \ll N_{0} U \ll \hbar \omega_{\|}$under the experimental conditions of interest, we can write the threedimensional wave function of the condensate as a product of a single-particle ground-state wave function in the parallel direction(s) and the (quasi)condensate wave function in the perpendicular direction(s). If we substitute this product wave function into the Gross-Pitaevskii equation and integrate out the parallel direction(s), we arrive at an effective equation for the (quasi)condensate wave function. Because of the abovementioned inequality, we can subsequently solve this equation using the Thomas-Fermi or local-density approximation [12]. To quantify the differences between the (quasi)condensate wave function and the single-particle ground-state wave function, we define a dimensionless coupling constant $g$ both in the noninteracting and in the interacting case. The first parameter we call the bare coupling constant $g_{\mathrm{B}}=U_{\mathrm{B}} / t_{\mathrm{B}}$ and it is calculated with the single-particle ground-state wave function in every site. The second parameter we call the renormalized coupling constant $g_{\mathrm{R}}=U_{\mathrm{R}} / t_{\mathrm{R}}$ and it is calculated using the single-particle ground state in the parallel direction(s) and a Thomas-Fermi density profile in the perpendicular direction(s). Because we have already included the on-site interaction effects in this coupling constant, we can now write down a renormalized single-band BoseHubbard model for the total optical lattice, where the creation and annihilation operators $\hat{a}_{i}^{\dagger}$ and $\hat{a}_{i}$, respectively, and the number operator $\hat{n}_{i}$ are not associated with the Wannier states of atoms in the lattice, but with the macroscopic wave function of the (quasi)condensate in each site. In particular, we have

$$
\hat{H}=-t_{\mathrm{R}} \sum_{\langle i, j\rangle} \hat{a}_{i}^{\dagger} \hat{a}_{j}+\frac{U_{\mathrm{R}}}{2} \sum_{i} \hat{n}_{i}\left(\hat{n}_{i}-1\right)-\mu_{\mathrm{R}} \sum_{i} \hat{n}_{i},
$$

where $\mu_{\mathrm{R}}$ is the effective chemical potential. The interaction parameter is given by $U_{\mathrm{R}}=\partial^{2} F^{\mathrm{os}} /\left.\partial N^{2}\right|_{N=N_{0}}$ $\equiv \partial \mu^{\mathrm{os}} /\left.\partial N\right|_{N=N_{0}}$, where $F^{\mathrm{os}}$ is the on-site free energy and $\mu^{\mathrm{os}}$ is the on-site chemical potential. Formally, the effective chemical potential is given by $\mu_{\mathrm{R}}=\mu-\mu^{\mathrm{os}}-U_{\mathrm{R}} / 2$, where the last term is substracted from the interaction energy to make the analogy to the single-band Bose-Hubbard model of Refs. [4,5] complete.

It is important to understand that the hopping term only describes the hopping between the (quasi)condensates in neighboring sites. While it is clear that this is a very good approximation in the case of neighboring condensates, it may not be immediately obvious in the case of neighboring quasicondensates. However, it should be noted that the effect of the hopping is large only when the system is in the superfluid phase, in which case all the sites couple to form a true threedimensional condensate. The tunneling strength can be calculated in the tight-binding limit and depends only on the overlap in the parallel direction(s). As a result the bare and renormalized values of $t$ are equal. However, the interaction energy is strongly reduced due to the repulsive on-site interactions that spreadout the (quasi)condensate wave function considerably. We find for a two-dimensional gas that

$$
g_{\mathrm{R}}=g_{\mathrm{B}}\left(\frac{\pi}{2}\right)^{1 / 4}\left(\frac{\ell_{\|}}{N_{0} a}\right)^{1 / 2} \propto g_{\mathrm{B}}\left(\frac{\ell_{\perp}}{R_{\mathrm{TF}}}\right)^{2}
$$

and for a one-dimensional gas that

$$
g_{\mathrm{R}}=g_{\mathrm{B}}\left(\frac{\pi}{2}\right)^{1 / 2}\left(\frac{\ell_{\|}^{2}}{3 N_{0} a \ell_{\perp}}\right)^{1 / 3} \propto g_{\mathrm{B}}\left(\frac{\ell_{\perp}}{R_{\mathrm{TF}}}\right) .
$$

Here $a$ is the positive $s$-wave scattering length of the atoms, $\ell_{\|}=\sqrt{\hbar / m \omega_{\|}}$and $\ell_{\perp}=\sqrt{\hbar / m \omega_{\perp}}$ are the harmonic-oscillator lengths in the parallel and perpendicular directions, respectively, and $R_{\mathrm{TF}}$ is the Thomas-Fermi radius of the (quasi)condensate. The physical interpretation of Eqs. (2) and (3) is that as a result of the repulsive interatomic interactions, the (quasi)condensate reduces its total energy by increasing its size in the perpendicular direction(s). This can be seen from the fact that the decrease in the coupling constant is inversely proportional to the increase in the surface or length of the twoor one-dimensional gas, respectively. Note that this reduction is particularly important for the critical conditions, which can be written as $g_{\mathrm{R}}>4 z N_{0}$ for large $N_{0}$ [5]. In order to verify the consistency of our two-step approach, we explicitly check the relevant energy scales using our results of Eqs. (2) and (3). First, we assumed that the mean-field interaction energy $N_{0} U_{\mathrm{R}}$ is much smaller than the trapping frequency in the parallel direction(s) $\hbar \omega_{\|}$. This requires that for a onedimensional lattice

$$
N_{0} \ll\left(\frac{\hbar \omega_{\|}}{\hbar \omega_{\perp}}\right)^{2} \sqrt{2 \pi} \frac{\ell_{\|}}{a} .
$$

Second, we also assumed that the crossover temperature for the formation of a (quasi)condensate in two dimensions is much lower than $\hbar \omega_{\|}$. This results in

$$
\hbar \omega_{\perp}\left(\frac{N}{\zeta(2)}\right)^{1 / 2} \ll \hbar \omega_{\|},
$$

where $N$ is the total number of atoms at every site. For typical numbers used in the experiments by Orzel et al. [6], we 


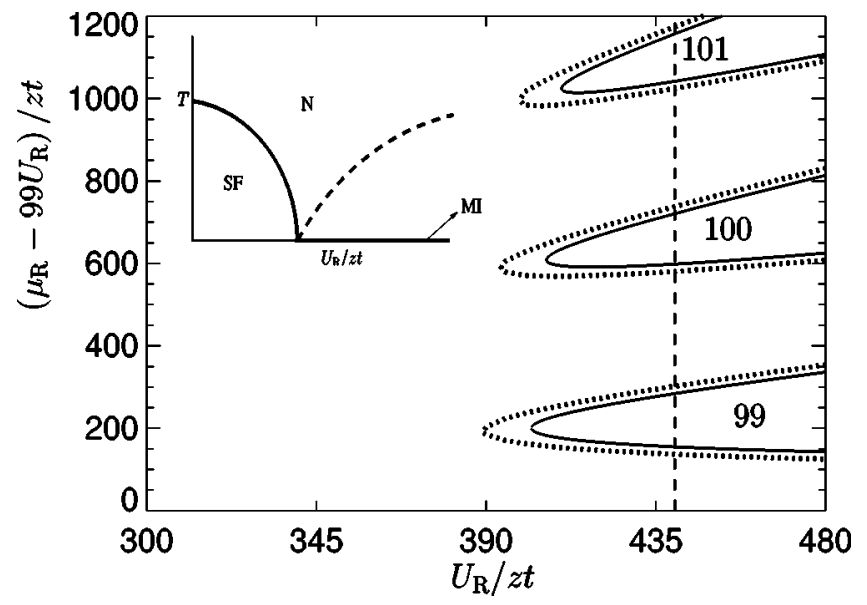

FIG. 1. Phase diagram of the Bose-Hubbard model in terms of the dimensionless chemical potential $\mu_{\mathrm{R}} / z t$ and the dimensionless coupling constant $U_{\mathrm{R}} / z$ t. The solid and dotted lines correspond to $T=0$ and $T=0.1 U_{c}$ (where $U_{c}$ is the critical $U_{\mathrm{R}}$ for the $N_{0}=100$ lobe), respectively. The inset shows a qualitative phase diagram in terms of the temperature $T$ and the coupling constant $g . N, \mathrm{SF}$, and MI indicate the normal gas phase, the superfluid, and the Mottinsulating phase, respectively.

find the condition $N_{0} \leqslant N \ll 10^{5}$, which means that our assumptions are valid for even the largest filling factor reported. For the case of the two-dimensional lattice of Greiner et al. [7], a similar inequality can be derived. It is found that this experiment is also in the regime where our assumptions are valid. Note that the use of the Thomas-Fermi approximation also imposes a lower limit on the filling factor, namely, $N_{0} a \gg l_{\perp}$. However, when the filling factor is below this limit, we are in the regime where we can safely use the bare coupling constant. We thus conclude that depending on the filling factor, either our renormalized or the bare theory is applicable to these experiments.

\section{THERMAL EFFECTS}

Besides the effect of thermal fluctuations on the number of (quasi)condensate atoms per site $N_{0}(T)$, which is accounted for by the equation of state of the low-dimensional Bose gas [11], there is also the effect of thermal fluctuations on the renormalized single-band Bose-Hubbard model itself. These thermal fluctuations are also present in a lattice with low filling factor. To study these thermal fluctuations, we use an on-site mean-field Hamiltonian that we can derive from Eq. (1), using the approach presented in Ref. [5]. We decouple the tunneling term, by introducing a complex meanfield parameter $\psi$ as follows: $\hat{a}_{i}^{\dagger} \hat{a}_{j}=\psi \hat{a}_{j}+\hat{a}_{i}^{\dagger} \psi^{*}-|\psi|^{2}$. Physically, $\psi$ is the superfluid order parameter that we choose to be real in the following. Performing the above substitution, we find

$$
\hat{H}(\psi)=-z t \psi\left(\hat{a}^{\dagger}+\hat{a}\right)+\frac{U_{\mathrm{R}}}{2} \hat{n}(\hat{n}-1)-\mu_{\mathrm{R}} \hat{n}+z t \psi^{2},
$$

with $z$ being the coordination number. Since this is an on-site Hamiltonian, we have dropped the site indices for simplicity.
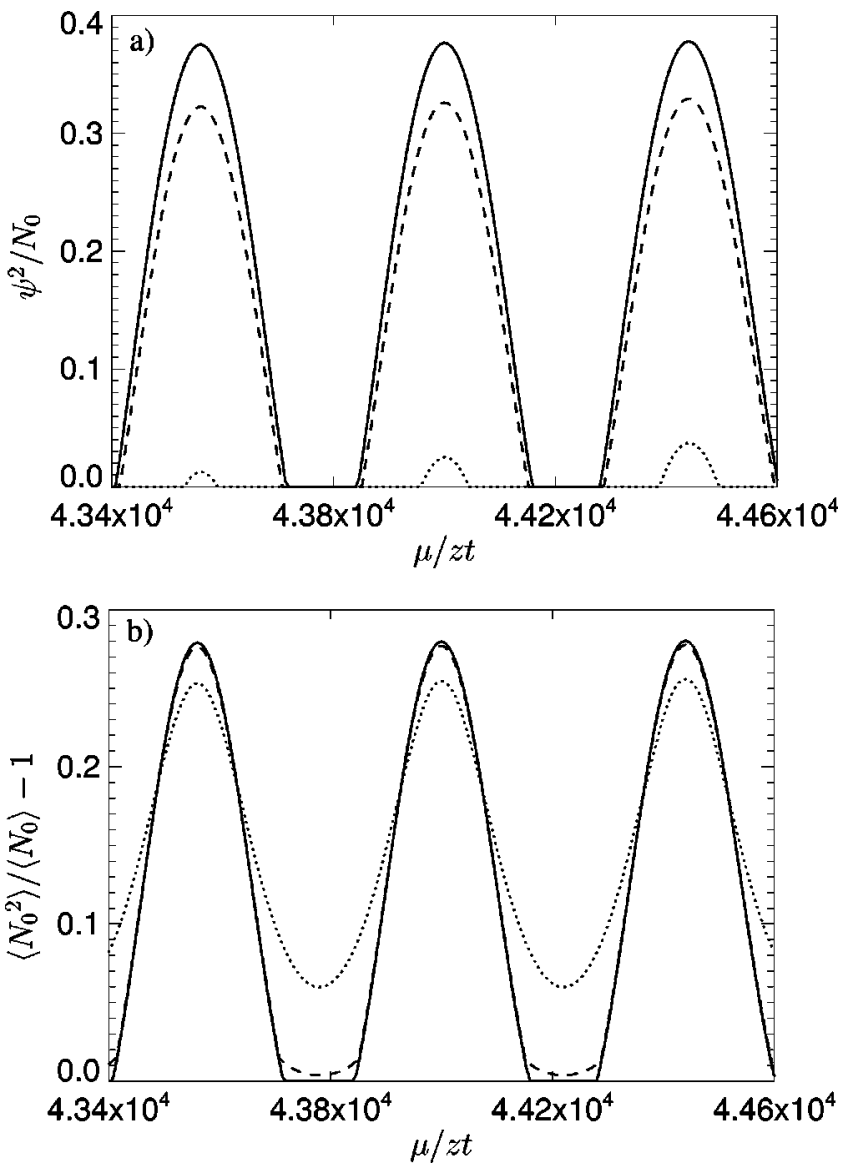

FIG. 2. Condensate fraction (a) and particle number fluctuations (b) as functions of the dimensionless coupling constant, for $k_{B} T$ $=0,0.08 U$, and $0.145 U$ (solid, dashed, and dotted lines, respectively). The coupling constant: $U_{\mathrm{R}} / z t=440$ (the dashed line in Fig. 1).

Moreover, we assumed that the chemical potential is chosen such that the expectation value of the number operator $\hat{n}$ is equal to the number of quasi(condensate) particles $N_{0}(T)$ in every site.

The zero-temperature phase diagram of this mean-field theory can be solved exactly $[5,10]$ and is shown in Fig. 1, where the Mott-insulator phases correspond to the various lobes. For nonzero temperatures the model can no longer be solved analytically and we have to resort to numerical methods. If we put $\psi=0$, we find that the eigenstates of Eq. (6) are given by number states. Using a basis consisting of these number states, running from a certain minimum filling factor $N_{\min }$ to a certain maximum $N_{\max }$, we can calculate the grand-canonical partition function $Z(\psi)=\operatorname{Tr}\left(e^{-H(\psi) / k_{B} T}\right)$ by diagonalizing the mean-field Hamiltonian given in Eq. (6). Next we determine the thermodynamic potential $\Omega(\psi)=$ $-k_{\mathrm{B}} T \ln Z(\psi)$ as a function of the order parameter $\psi$. For zero temperature the calculation converges when $N_{\max } \simeq N_{0}$ +4 and $N_{\min } \simeq N_{0}-4$, where $N_{0}$ is the filling factor of the relevant Mott-insulator lobe. For nonzero temperatures, more states must be included.

To obtain the relevant thermodynamic quantities, we minimize the grand potential $\Omega(\psi)$ and the value of $\psi$ at the 
minimum of $\Omega(\psi)$ corresponds physically to the square root of the number of atoms that is superfluid in the direction(s) parallel to the periodicity of the lattice. In the Mott insulator, the gas is only insulating in the direction(s) parallel to the periodicity of the optical lattice, whereas it is always a superfluid in the perpendicular direction(s). The other quantity of interest is the value of the number fluctuations. This number is important because in the Mott-insulator phase the number fluctuations are exactly zero.

The final results of the calculations are shown in Fig. 2. In these figures, the longitudinal superfluid fraction and the number fluctuations are plotted along the dashed line in Fig. 1 for different temperatures. It can clearly be seen from Fig. 2(a) that the superfluid part of the phase diagram decreases with increasing temperature. In addition Fig. 2(b) shows that at zero temperature the density fluctuations drop exactly to zero in the Mott-insulating regions, but this does not happen at nonzero temperature. This is a result of the fact that the superfluid to Mott insulator transition is a quantum phase transition. The reason that there is still a reduction in particle-number fluctuations at nonzero temperature is that the excitation spectrum of a fluctuation is gapped in this region [5], which means that the fluctuations are exponentially suppressed. Due to this strong suppression of the number fluctuations, one will be able to observe a phase that is formally not a Mott insulator, but experimentally has very similar features. Another feature we can clearly see in Fig. 2(b) is that the part of the phase diagram where the number fluctuations are suppressed also decreases with increasing temperature, and shrinks in the opposite direction of that of the superfluid part.

On the basis of the above calculations, we can draw the nonzero temperature phase diagram shown in Fig. 1. In this figure, the solid lines indicate the superfluid to Mott insulator transition at zero temperature and the dotted lines indicate the superfluid to normal transition at nonzero temperature. The inset shows the phase diagram in terms of the temperature and the coupling constant. This diagram agrees very well with the general description given by Sachdev [10].

\section{CONCLUSION}

We have shown that for low-dimensional lattices, which generally have a filling factor much larger than 1, we should, in principle, solve a many-band Bose-Hubbard model. This can be achieved by first solving the on-site many-body problem, and then deriving an effective theory that describes the coupling between the sites in the optical lattice in terms of a renormalized single-band Hubbard model. We have calculated the effects of thermal excitations in this renormalized model and we have shown that the number fluctuations in the above model can only drop to zero in the absence of thermal fluctuations. However, if the temperature is sufficiently low, the number fluctuations are exponentially suppressed. This means that at a certain nonzero temperature, the crossover to the Mott-insulator phase can still be observed if the coupling constant is increased to a value larger than the zerotemperature critical value (cf. Fig. 1). It is important to realize that to experimentally obtain the Mott insulator with a large filling factor $N_{0}$, the coupling constant $g_{\mathrm{R}}=U_{\mathrm{R}} / t$ must be larger than $4 z N_{0}$. However, Eqs. (2) and (3) show that the renormalized coupling constant is much smaller than the bare coupling constant for a low-dimensional optical lattice. We therefore conclude that in terms of the bare coupling constant, which is the experimentally relevant control parameter, the Mott-insulator phase is much more difficult to obtain than is naively anticipated on the basis of a purely single-band Bose-Hubbard model.
[1] M.R. Andrews, C.G. Townsend, H.-J. Miesner, D.S. Durfee, D.M. Kurn, and W. Ketterle, Science 275, 637 (1997).

[2] B.P. Anderson and M.A. Kasevich, Science 282, 1686 (1998).

[3] M. Greiner, O. Mandel, T. Esslinger, T.W. Hänsch, and I. Bloch, Nature (London) 415, 39 (2002).

[4] D. Jaksch, C. Bruder, J.I. Cirac, C.W. Gardiner, and P. Zoller, Phys. Rev. Lett. 81, 3108 (1998).

[5] D. van Oosten, P. van der Straten, and H.T.C. Stoof, Phys. Rev. A 63, 053601 (2001).

[6] C. Orzel, A.K. Tuchman, M.L. Fenselau, M. Yasuda, and M.A. Kasevich, Science 291, 2386 (2001).

[7] M. Greiner, I. Bloch, O. Mandel, T.W. Hänsch, and T.
Esslinger, Phys. Rev. Lett. 87, 160405 (2001).

[8] J. Javanainen, Phys. Rev. A 60, 4902 (1999).

[9] K. Burnett, M. Edwards, C.W. Clark, and M. Shotter, J. Phys. B 35, 1671 (2002).

[10] S. Sachdev, Quantum Phase Transitions (Cambridge University Press, Cambridge, 2001).

[11] J.O. Andersen, U. Al Khawaja, and H.T.C. Stoof, Phys. Rev. Lett. 88, 070407 (2002); U. Al Khawaja, J.O. Andersen, N.P. Proukakis, and H.T.C. Stoof, Phys. Rev. A 66, 013616 (2002).

[12] F. Dalfovo, S. Giorgini, and L.P. Pitaevskii, Rev. Mod. Phys. 71, 463 (1999). 